\title{
Louise Iseult-Paradis
}

Anthropologue, département d'anthropologie, Université de Montréal

(1979)

\section{"L’archéologie, \\ pourquoi, comment?”}

Un document produit en version numérique par Jean-Marie Tremblay, bénévole, professeur de sociologie au Cégep de Chicoutimi

Courriel: jean-marie tremblay@uqac.ca

Site web pédagogique : http://www.uqac.ca/jmt-sociologue/

Dans le cadre de la collection: "Les classiques des sciences sociales"

Site web: http://www.uqac.ca/Classiques_des_sciences_sociales/

Une collection développée en collaboration avec la Bibliothèque

Paul-Émile-Boulet de l'Université du Québec à Chicoutimi

Site web: http://bibliotheque.uqac.ca/ 
Cette édition électronique a été réalisée par Jean-Marie Tremblay, bénévole, professeur de sociologie au Cégep de Chicoutimi à partir de l'article de :

Louise Iseult-Paradis

Anthropologue, département d'anthropologie, Université de Montréal

“L’archéologie, pourquoi, comment?” (1979)”

Un article publié dans l'ouvrage collectif intitulé: Perspectives anthropologiques. Un collectif d'anthropologues québécois, chapitre 4, pp. 39 à 57. Montréal: Les Éditions du Renouveau pédagogique, 1979, 436 pp.

[Autorisation formelle accordée, le 17 octobre 2005, par Madame Louise Iseult-Paradis de diffuser tous ses travaux de recherche et toutes ses publications.]

Courriel louise.i.paradis@umontreal.ca

Polices de caractères utilisée :

Pour le texte: Times New Roman, 14 points.

Pour les citations : Times New Roman 12 points.

Pour les notes de bas de page : Times New Roman, 12 points.

Édition électronique réalisée avec le traitement de textes

Microsoft Word 2004 pour Macintosh.

Mise en page sur papier format

LETTRE (US letter), 8.5' x 11'’)

Édition complétée le 12 novembre 2005 à Chicoutimi, Ville de Saguenay, province de Québec. 


\section{Louise Iseult-Paradis}

Anthropologue, département d'anthropologie

Université de Montréal (1978)

\section{“L’archéologie, pourquoi, comment?”}

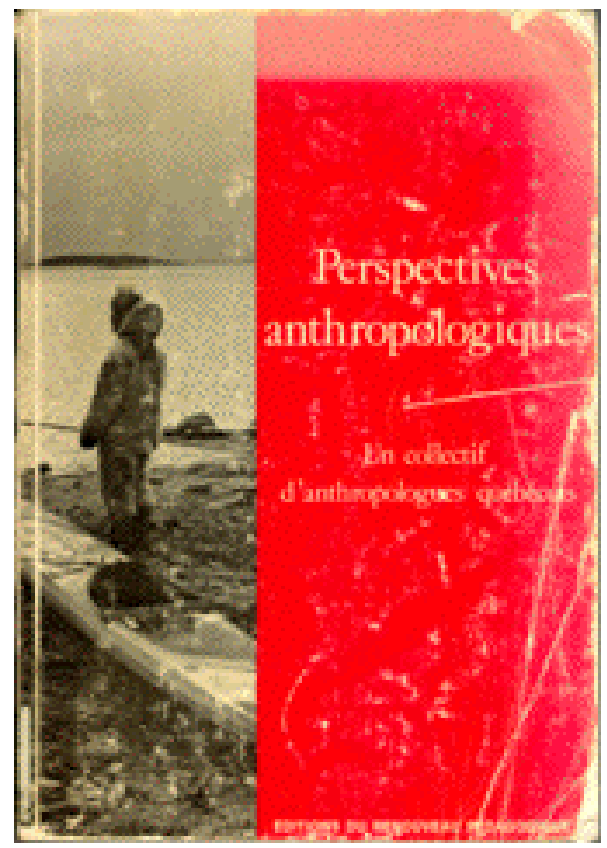

Un article publié dans l'ouvrage collectif intitulé: Perspectives anthropologiques. Un collectif d'anthropologues québécois, chapitre 4, pp. 39 à 57. Montréal: Les Éditions du Renouveau pédagogique, 1979, 436 pp. 


\section{Table des matières}

$\underline{\text { Introduction }}$

1. Définition et spécificité de l'archéologie

2. $\quad$ Principales étapes du développement de la discipline

3. Une esquisse de la préhistoire des sociétés

Le Pléistocène : sociétés prédatrices

Le post-pléistocène: sociétés productrices

Bilan

4. $\quad$ Pratique de l'archéologie

Demander - observer - décrire

Analyser, associer, interpréter, répondre

Teotihuacán

Figure 4.1 Teotihuacán : pyramide du soleil et avenue des Morts.

Figure 4.2 Carte de Teotihuacan. Tiré de Urbanization at Teotihuacan, Mexico, v. 1, The Teotihuacan Map, 1973 by René Millon, tous droits réservés.

Figure 4.3 Teotihuacán : pyramide de la lune.

Figure 4.4 Teotihuacán : résidences près de la pyramide du soleil.

Figure 4.5 Teotihuacan : masques et figurines de pierre. (American Museum of Natural History).

Conclusion

Bibliographie 
Louise Iseult Paradis

Université de Montréal

“L'archéologie, pourquoi et comment ?”

Un article publié dans l'ouvrage collectif intitulé : Perspectives anthropologiques. Un collectif d'anthropologues québécois, chapitre 4, pp. 39-57. Montréal : Les Éditions du Renouveau pédagogique, 1979, 436 pp.

\section{Introduction}

$\underline{\text { Retour à la table des matières }}$

L'homme s'est partout et toujours interrogé sur lui-même, sur son origine et sur sa relation à l'univers. En fait, objectiver et conceptualiser la réalité, à travers les symboles du langage, distinguent l'espèce humaine du reste du monde vivant. Toute société possède son propre système d'explication de la réalité, qui trouve son expression dans la mythologie, la religion, la philosophie, la science, etc.

L'archéologie se range parmi ces disciplines qui cherchent à expliquer le phénomène humain, son unité et sa diversité. Sa perspective est diachronique autant que synchronique et ce sont des réponses « scientifiques » qu'elle entend apporter à son enquête.

Dans ce texte, j'aimerais introduire le lecteur à ce qu'est l'archéologie. Dans un premier temps, j'examinerai le caractère spécifique de 
cette discipline. Ensuite, à travers l'historique de son développement comme science et une esquisse de son apport à la connaissance des sociétés préhistoriques, je tenterai d'illustrer le cheminement théorique qu'a parcouru cette jeune discipline. Mon examen portera enfin sur la pratique même de l'archéologie ; la description d'un site archéologique complétera cette courte introduction à l'archéologie.

\section{1. \\ Définition et spécificité de l'archéologie}

$\underline{\text { Retour à la table des matières }}$

Comment définir de façon plus précise l'archéologie ? Je retiendrai deux points : l'objet spécifique et la dimension temporelle dans laquelle s'inscrit l'archéologie.

En premier lieu, l'archéologie se distingue par son objet d'étude : l'étude des sociétés par le biais des témoins matériels des activités et des comportements qu'elles ont laissés derrière elles.

Lorsque des documents écrits ou des témoignages oraux existent pour la période étudiée, l'archéologie en tient évidemment compte. En général toutefois, ceux-ci sont absents, mais, dans les lieux où les populations étudiées ont vécu, des traces d'activités de toutes sortes (subsistance, interactions diverses, traitement des morts, etc.) témoignent de leur existence. Il est étonnant de voir combien riches et révélateurs sont les indices de ces comportements, tels que : outils de pierre, d'ossements et d'andouillers, poterie, vannerie, tissage, tombes et cimetières, maisons et palais, restes végétaux et animaux. Parmi ces indices, certains, grandioses, se révèlent de précieux objets de collection, mais la plus grande partie présente un aspect beaucoup moins remarquable ; tous, et au même titre, sont importants pour l'archéologue. 
Par rapport à la totalité de la culture étudiée, les données dont dispose l'archéologue se limitent donc, presque exclusivement, à des témoins matériels d'activités. A ce niveau même, l'information que livrent les vestiges rencontrés au cours de reconnaissances ou d'excavations est variable et fragmentaire. Seuls certains éléments de l'inventaire culturel parviennent jusqu'à nous. La pierre taillée (outils et structures) et la céramique sont sûrement les matériaux les plus résistants aux temps et aux agents naturels ; ils constituent, de ce fait, les indices les plus fiables et les plus communément étudiés par l'archéologue. Dans de bonnes conditions de préservation, telles que dans des milieux arides ou dans des sols anaérobies ${ }^{1}$ il arrive que d'autres catégories de vestiges soient conservées : bois, cuir, fibres végétales, tissus, vannerie, graines et ossements. Compte tenu de ces restrictions, c'est à partir de ces indices, de leur signification propre et des associations entre eux que l'archéologie reconstruit l'histoire des sociétés, qu'elle tente d'expliquer les causes des adaptations culturelles et les mécanismes selon lesquels elles opèrent. À cette fin, elle a mis au point un ensemble d'outils techniques et méthodologiques, ce qui, d'ailleurs, la distingue le mieux des autres disciplines. J'y reviendrai lorsqu'il sera question de la pratique de l'archéologie.

Au point de vue terminologique, une remarque s'impose ici. A l'origine, le terme « archéologie » ne faisait référence qu'à l'ensemble de techniques développées par la discipline. Dans la tradition américaine toutefois, et j'adopte ici cette définition, ce terme recouvre autant l'aspect théorique que technique de la discipline et comprend l'étude des sociétés, de leur histoire et de leur évolution, par le biais des indices matériels de leur comportement. C'est pourquoi l'on parle d'archéologie préhistorique, d'archéologie historique, d'archéologie industrielle, etc.

En second lieu, l'archéologie s'inscrit dans une dimension temporelle déterminée. De par son objet même d'étude, cette discipline couvre toute la préhistoire et l'histoire des sociétés. Elle détient la responsabilité exclusive de l'étude de la préhistoire, c'est-à-dire des sociétés

Sol anaérobie : type de soi compact contenant peu d'oxygène et favorisant de ce fait la conservation des témoins culturels qui y sont enfouis. 
antérieures à l'apparition de l'écriture. Néanmoins, elle contribue de plus en plus aujourd'hui à la connaissance des sociétés historiques et modernes.

L'archéologie n'est pas la seule discipline à toucher aux problèmes d'origine et de développement des sociétés humaines. Des sciences comme la géologie, la paléobotanique et la paléozoologie y contribuent également. Ces trois disciplines ont en commun d'étudier le passé lointain. de la terre et des êtres vivants. La géologie étudie les transformations successives qu'a connues la terre depuis son origine. Son domaine d'étude s'étend beaucoup plus loin dans le temps que celui de l'archéologie, la formation de la terre étant estimée à environ six milliards d'années. La paléobotanique étudie les transformations subies par les végétaux au cours du temps à l'aide des restes végétaux microscopiques et fossiles contenus dans les couches terrestres. Cette discipline, relativement jeune, permet de connaître l'environnement végétal qui existait sur terre à une époque donnée et nous donne des indications précieuses sur le climat qui y prévalait. La paléozoologie étudie l'évolution des différentes espèces animales à partir de leurs restes osseux conservés sous forme fossilisée.

Ces sciences sont indispensables à l'archéologie, car elles permettent de situer l'environnement physique dans lequel sont apparues et se sont développées les premières sociétés humaines. Toutefois, l'espèce humaine n'intervenant que récemment dans le système écologique, ces sciences ne touchent qu'indirectement à l'étude des sociétés humaines.

À l'autre extrémité du continuum temporel, l'histoire partage avec l'archéologie un intérêt pour l'évolution des sociétés humaines. L'historien connaît le passé à travers les documents écrits, l'archéologue, à travers les témoignages des vestiges matériels. L'histoire traite du passé récent des sociétés humaines, les documents écrits n'existant que depuis quelques millénaires. La plus grande partie de l'histoire de l'humanité n'a pas été écrite, le témoignage des vestiges matériels constituant la seule preuve de son existence. C'est à l'archéologue que revient la tâche de reconstituer le passé des sociétés humaines en étudiant les vestiges matériels que des conditions naturelles favorables ont préservés. L'archéologie préhistorique étudie les vestiges matériels laissés par la plupart des sociétés humaines, jusqu'au moment de l'in- 
vention de l'écriture. Elle utilise alors d'une manière exclusive le témoignage des vestiges matériels. On parle d'archéologie historique, ou même industrielle, quand l'archéologue aborde l'étude de sociétés pour lesquelles il existe des documents écrits : par exemple, les situations de contact en Nouvelle-France et en Nouvelle-Espagne. L'archéologie devient alors complémentaire de l'histoire bien que les perspectives propres à ces deux disciplines soient distinctes.

Quant à la philosophie, elle analyse sensiblement les mêmes aspects de la réalité que l'archéologie. Elle se pose les mêmes questions sur le phénomène humain mais utilise d'autres modèles pour tenter de l'expliquer. Elle s'intéresse prioritairement à l'analyse des lois du fonctionnement de l'esprit, mais trop souvent à partir d'une vision très ethnocentrique de l'univers. Ce qui la distingue surtout de l'archéologie, c'est son objet spécifique d'étude, les idées et les concepts, qui ne sont certes pas directement observables par l'archéologue.

Parmi tant d'autres, les disciplines mentionnées ici partagent avec l'archéologie un intérêt pour l'étude de l'homme et de son évolution. Elles en diffèrent cependant par leur objet spécifique d'étude, leur orientation théorique et leurs méthodes d'approche de la réalité sociale.

\section{2. \\ Principales étapes du développement de la discipline}

$\underline{\text { Retour à la table des matières }}$

L'archéologie est une discipline jeune. Il y a à peine plus de cent ans, la conception de l'antiquité de l'homme qui prédominait en Europe tenait en grande partie du mythe. Elle était, en effet, fortement marquée par l'interprétation que l'on faisait alors des dogmes chrétiens. L'étude des Saintes Écritures avait même permis de fixer à une date précise - en l'an 4004 av. J.-C. - l'apparition, la création, devrais- 
je dire, du premier être vivant. Cela représente, à peu de chose près, l'ensemble des renseignements qu'aurait pu comporter un manuel de préhistoire de cette époque. L'étude du passé de l'homme commençait à cette date et relevait davantage de l'histoire que de la préhistoire.

L'histoire du développement de l'archéologie témoigne de l'évolution rapide des idées dans cette discipline. Elle s'insère dans un courant scientifique beaucoup plus vaste, cependant, qui, tout au long de son cheminement, en a teinté les principes et les interprétations.

C'est à partir du milieu du 19e siècle que se développent la théorie évolutionniste et les lois géologiques d'uniformité et de superposition. Elles créent une véritable révolution scientifique en Europe. L'évolutionnisme biologique, grâce aux travaux de Darwin, dévoile le mécanisme qui permet d'expliquer l'histoire et la variabilité du monde végétal et animal : la sélection naturelle. En géologie, avec la loi d'uniformité, on rejette l'idée de catastrophisme et accepte celle de la régularité dans la formation et la déposition des couches terrestres. La loi de superposition précise l'ordre de déposition : dans une pile d'unités de déposition dans laquelle le haut et le bas peuvent être identifiés, l'ordre de succession de bas en haut donne l'ordre de déposition. C'est au sein de ce courant scientifique que l'anthropologie et l'archéologie moderne ont fait leurs premières armes. Pour l'anthropologie, c'est la grande époque de l'évolutionnisme culturel, qui explique l'évolution culturelle par le passage des sociétés par des stades successifs de développement (barbarie, sauvagerie et civilisation). Pour l'archéologie préhistorique, c'est la possibilité de se mettre en quête de la véritable antiquité de l'espèce humaine et de trouver des traces de stades antérieurs de développement culturel. Les lois géologiques d'uniformité et de superposition lui permettent de valider ses trouvailles.

Au début du 20e siècle, en réaction contre le manque de données empiriques appuyant les énoncés des théories évolutionnistes et contre la rigidité de ces théories, les préhistoriens s'engagent dans une période d'intense exploration archéologique. Ils s'appliquent à découvrir des sites, à faire des classifications, à élaborer des typologies et à établir des séquences chronologiques. Les techniques de classification se 
développent et l'avènement de la datation au radiocarbone (1950) ${ }^{2}$ couronne cette période durant laquelle la forme, le temps et l'espace constituent les thèmes centraux de la recherche archéologique. Des sites préhistoriques sont identifiés sur presque tous les continents : on en connaît la date, la distribution géographique et le contenu. Migration et diffusion deviennent les mécanismes explicatifs de l'histoire culturelle. Cette tendance théorique est la conséquence directe de l'orientation qu'a prise la recherche anthropologique et archéologique de cette époque. À partir de la masse de données archéologiques et anthropologiques recueillie, on établit de véritables cartes de distribution de traits culturels à travers le monde. Les sociétés sont perçues comme des ensembles de traits culturels. Le degré de similarité ou de différence entre ces cultures est mesuré à partir du nombre de traits partagés. Il n'y avait qu'un pas à faire pour passer de la description à l'explication et on l'a franchi : tout pouvait et devait s'expliquer par la diffusion ou la migration.

Ces approches méthodologiques et théoriques créent beaucoup d'insatisfaction parmi les chercheurs. Tout comme en anthropologie où, après une période d'intense cueillette de données, on tente de les expliquer, l'archéologie se tourne vers l'interprétation, vers la compréhension des dynamismes sociaux. On veut savoir comment et pourquoi - et non plus seulement quand et où - sont apparues la production alimentaire, les villes, l'écriture, les formes d'organisation sociale et politique, etc. Jusque-là indices de changement chronologique, les témoins archéologiques se voient attribués une nouvelle fonction, soit celle de servir d'indices de comportements sociaux. Cette tendance entraîne un réaménagement, tant aux niveaux de la pratique que de la théorie archéologique. L'archéologie entre dans l'ère de la multidisciplinarité ; elle sollicite l'aide de disciplines connexes pour combler ses lacunes au niveau de la cueillette des données et de leur interprétation. Des palynologues ${ }^{3}$, des zoologues, des géologues, des pédologues ${ }^{4}$,

2 Technique permettant de dater les vestiges archéologiques, à l'aide des éléments radioactifs (isotope carbone 14) présents dans toute matière organique, trouvée en association avec une couche culturelle.

3 Palynologue : spécialiste de l'étude du pollen et des spores.

4 Pédologue : spécialiste des sois. 
des climatologues ${ }^{5}$, et j'en passe, sont recrutés pour faire partie des équipes d'archéologie. L'utilisation des mathématiques et des ordinateurs dans le traitement des données ainsi que de la chimie et de la physique dans la datation des vestiges et l'identification des sources de matières premières constituent des exemples importants de cette collaboration de diverses disciplines à l'archéologie. Au plan théorique enfin, pour expliquer certains phénomènes sociaux, l'archéologie fait appel à des modèles empruntés à d'autres disciplines, notamment à l'analyse locationnelle en géographie, à la théorie des systèmes et à des modèles écologiques.

La tendance est aujourd'hui à la spécialisation en science, et l'archéologie n'échappe pas à la règle. Ces développements récents mettent l'archéologie en situation de crise : il s'agit pour elle de réduire le décalage entre les questions posées et les méthodes aujourd'hui à sa disposition pour y répondre. Il s'agit également d'intégrer à l'archéologie les diverses contributions apportées par les autres disciplines afin de pouvoir ainsi les rendre opératoires.

\section{3. \\ Une esquisse de la préhistoire des sociétés}

$\underline{\text { Retour à la table des matières }}$

L'archéologie peut se vanter d'avoir fait jaillir la lumière sur la préhistoire des sociétés humaines. À la question fondamentale qu'elle se pose, soit " qu'est-ce que l'homme ? ", elle est aujourd'hui en mesure d'offrir quelques réponses.

L'origine de l'humanité remonte à plus de trois millions d'années. Elle pourrait se résumer en un long processus d'expansion, d'adapta-

5 Climatologue : spécialiste du climat. 
tion et de diversification, tant sur le plan biologique que culturel (voir Tableau 4.1).

\section{Le Pléistocène : sociétés prédatrices}

$\underline{\text { Retour à la table des matières }}$

C'est durant la période géologique du Pléistocène, qui s'étend d'environ trois millions d'années jusqu'à 10000 ans av. J.-C., qu'apparaissent les premières populations humaines. Les australopithèques, premiers groupes considérés comme hominidés, occupaient les régions tropicales et subtropicales de l'Afrique. Ils étaient constitués en petites bandes nomades et vivaient dans des campements temporaires, se nourrissant des produits de la chasse et de la cueillette. Leur équipement se composait d'outils rudimentaires en pierre, principalement des galets dont ils dégageaient un ou deux éclats.

Peu à peu, de nouveaux territoires furent occupés par des populations d'hominidés ressemblant de plus en plus à l'homme moderne, et qui s'établirent ainsi progressivement en Afrique, en Asie et en Europe. Ces populations (Homo erectus, Homo sapiens) se sont d'abord adaptées à des environnements tropicaux et subtropicaux, puis aux régions tempérées et enfin aux régions plus froides des zones subarctiques et arctiques. Les derniers continents conquis furent l'Australie et les Amériques : les premières traces d'occupation, par des hommes biologiquement modernes (Homo sapiens sapiens), n'y remontent pas à plus de 50000 ans.

Intimement liée aux transformations biologiques et à l'exploitation de nouveaux espaces, l'évolution culturelle des populations humaines du Pléistocène va dans le sens d'une plus grande efficacité technologique et de l'accroissement de la diversité culturelle. C'est à un rythme très lent, au cours d'une période de trois millions d'années, que s'opèrent ces premières adaptations. 
Pendant toute cette époque, toutefois, et malgré l'accroissement de la diversité culturelle et sociale, ces populations ont en commun un même mode de production : ce sont des sociétés prédatrices. Par cela, je veux dire que la subsistance est basée sur l'acquisition de ressources végétales et animales. Que ce soit au moyen de la pêche, de la chasse, de la cueillette ou de la trappe, ces systèmes d'acquisition ne nécessitent pas de contrôle sur la production ou la reproduction de nourriture. Il semble bien que, malgré la variété d'adaptations sociales observées, ce mode de production n'ait pas offert les conditions propices à l'existence de sociétés étatiques ou urbaines. La limite imposée sur la dimension de la population qui pouvait exploiter efficacement un territoire et la tendance au nomadisme que demande généralement ce mode de production auraient sûrement été à l'encontre d'un tel développement. L'examen des données archéologiques de l'époque du Pléistocène permet d'inférer l'existence de bandes ou de tribus, de groupes généralement petits à l'intérieur desquels les signes de stratifications sont absents ou alors liés à une organisation clanique ou équivalente. 
Tableau 4.1

Esquisse de la préhistoire des sociétés humaines.

\begin{tabular}{|c|c|c|c|c|c|c|}
\hline $\begin{array}{l}\text { Années } \\
\text { avant le } \\
\text { présent }\end{array}$ & $\begin{array}{l}\text { Époque } \\
\text { géologique }\end{array}$ & $\begin{array}{l}\text { Évolution } \\
\text { Homini- } \\
\text { dés }\end{array}$ & Afrique & $\begin{array}{l}\text { Europe et Méditer- } \\
\text { ranée }\end{array}$ & Asie & Amérique \\
\hline 10000 & $\begin{array}{l}\text { Post- } \\
\text { pléistocène }\end{array}$ & 4 & & $\begin{array}{l}\text { Néolithique (civili- } \\
\text { sation) Mésolithique } \\
\text { (production alimen- } \\
\text { taire) }\end{array}$ & $\begin{array}{l}\text { (civilisation) } \\
\text { Hoabinhien }\end{array}$ & $\begin{array}{c}\text { (civilisation) } \\
\text { Formatif } \\
\text { (Production } \\
\text { alim.) } \\
\text { Archaïque }\end{array}$ \\
\hline $\begin{array}{l}50000 \\
100000\end{array}$ & $\begin{array}{l}\text { Pléistocène } \\
\text { récent }\end{array}$ & $\begin{array}{c}\text { H.S. } \\
\text { sapiens } \\
\text { Homo } \\
\text { sapiens }\end{array}$ & $\begin{array}{l}\text { Age de pierre } \\
\text { récent } \\
\text { Age de pierre } \\
\text { moyen }\end{array}$ & $\begin{array}{l}\text { Paléolithique } \\
\text { Supérieur } \\
\text { Paléolithique } \\
\text { Moyen : } \\
\text { Moustérien }\end{array}$ & $\begin{array}{c}? \\
\text { (mal défini) }\end{array}$ & Lithique \\
\hline $\begin{array}{l}500000 \\
1 \text { Million }\end{array}$ & $\begin{array}{l}\text { Pléistocène } \\
\text { moyen }\end{array}$ & $\begin{array}{l}\text { Homo } \\
\text { erectus }\end{array}$ & $\begin{array}{c}\text { Acheuléen } \\
\text { (industries de } \\
\text { bifaces) }\end{array}$ & $\begin{array}{l}\text { Paléolithique } \\
\text { Ancien : Acheuléen } \\
\text { clactonien }\end{array}$ & $\begin{array}{c}\text { Industries de } \\
\text { couperets et } \\
\text { de taillants }\end{array}$ & \\
\hline 2 Millions & $\begin{array}{c}\text { Pléistocène } \\
\text { ancien }\end{array}$ & $\begin{array}{l}\text { Australo- } \\
\text { pithèque }\end{array}$ & $\begin{array}{l}\text { Olduvai* } \\
\text { (industries } \\
\text { sur galets) }\end{array}$ & & & \\
\hline 3 Millions & & & & & & \\
\hline
\end{tabular}




\section{Le post-pléistocène : sociétés productrices}

$\underline{\text { Retour à la table des matières }}$

Le Pléistocène est une période durant laquelle l'hémisphère nord est le lieu de glaciations intenses. A la fin de cette période, un réchauffement général se produit, les glaciers disparaissent et de nouveaux espaces ressurgissent. On ne sait pas encore dans quelle mesure les changements climatiques et écologiques qui ont résulté de la fin des dernières grandes glaciations ont influé sur les importants changements sociaux que l'on observe alors. Après tout, même pendant le Pléistocène, de tels épisodes s'étaient produits. Quoi qu'il en soit, il y a environ 10000 ans, au Proche-Orient, on a commencé à cultiver le blé et l'orge et à domestiquer les bovins et les caprins. Quelque trois millénaires plus tard, la production du maïs, de la fève et de la courge débutait au Mexique et dans les Andes centrales. Ce sont là les premiers exemples bien documentés de la production alimentaire. L'homme assumait dès lors un contrôle sur la production et la reproduction des plantes et des animaux. Les conséquences de l'adoption de ce nouveau mode de production, bien que déterminantes, sont encore mal comprises. Elles se révèlent tant dans le domaine de la relation de l'homme avec son environnement (prédiction, sécurité, surplus) que dans celui de l'organisation des relations entre individus et groupes (sédentarité, concentration de plus grandes populations, redistribution de surplus, inégalité sociale). Et ce seront les sociétés agricoles qui produiront les premiers États.

À partir de ce moment, le rythme des transformations et diversifications culturelles et sociales s'accélère. La population mondiale s'accroît et occupe intensivement toutes les latitudes. Des innovations technologiques (poterie, métallurgie), de nouvelles formes sociales et de nouveaux modes d'établissement sont inaugurés. Ainsi, on voit naître et se développer les premières civilisations et l'urbanisme au Proche-Orient, en Inde, en Chine, en Mésoamérique et dans l'aire andine. Mais toutes les sociétés ne suivent pas un même cheminement. Au 
contraire, elles continuent de se diversifier avec leurs lois, leurs organisations économiques et politiques, leurs langues et leurs idéologies, leurs arts et leurs sciences. A côté des sociétés agricoles, on trouve des sociétés de chasseurs, de pêcheurs ou de pasteurs, ou encore un mélange de ces divers modes de subsistance.

\section{Bilan}

\section{$\underline{\text { Retour à la table des matières }}$}

Les grandes lignes de la préhistoire humaine commencent à peine à émerger ; de nombreuses questions restent sans réponse, ne serait-ce qu'en ce qui a trait à la reconstitution des événements. Mais, plus encore, on s'interroge pour comprendre les processus qui nous ont conduits aux formes de sociétés que l'on connaît aujourd'hui et pour expliquer les adaptations et les choix qui se sont faits depuis trois millions d'années. Par exemple, pourquoi les gens se sont-ils mis à cultiver ? Pourquoi les villes et pourquoi les dieux ? Comment expliquer que les hommes et les femmes en société soient à la fois si semblables et pourtant si différents ? C'est à ce genre de questions et dans cette perspective anthropologique que s'inscrit l'archéologie: connaître l'homme à travers les témoins qui restent de son histoire et de son évolution et grâce à eux.

\section{4. \\ Pratique de l'archéologie}

$\underline{\text { Retour à la table des matières }}$

Les diverses étapes logiques de l'enquête archéologique sont présentées schématiquement au tableau 4.2. L'importance accordée à chacune de ces étapes varie en fonction des objectifs particuliers à la recherche en cours. Dans la pratique toutefois, l'ordre logique est rarement respecté : en archéologie comme dans toute discipline scienti- 
fique, la méthodologie se caractérise par une succession de retours en arrière et de remises en question.

L'archéologie a mis au point un ensemble de techniques en vue de recueillir, d'enregistrer et d'analyser son matériel. C'est sur ce plan celui de la réalité qu'elle observe et des moyens qu'elle utilise pour l'appréhender - qu'elle se distingue des autres domaines de l'anthropologie. Je m'y arrêterai quelques instants.

\section{Demander - observer - décrire}

$\underline{\text { Retour à la table des matières }}$

Une fois l'hypothèse énoncée, les moyens de financement de la recherche assurés et les divers permis obtenus, l'archéologue se retrouve face à son univers d'analyse. A partir de témoins fragmentaires d'activités humaines, parfois apparents au niveau du sol, parfois estimés sous la surface terrestre ou aquatique, il tente de rassembler les pièces du casse-tête. Au départ, il dispose de deux données, soient les témoins matériels de comportements sociaux et l'environnement dans lequel ils ont été produits ou utilisés. La première tâche de l'archéologue consiste à identifier et à enregistrer ces données. C'est alors qu'il doit mettre à profit ses qualités d'observateur minutieux, de cartographe, de photographe, de mathématicien, de géologue et de devin. L'archéologue doit, avant tout, savoir prendre des décisions rapidement et dans plusieurs domaines simultanément. Certaines décisions, telles le choix des techniques d'échantillonnage et le mode d'enregistrement des données, peuvent être prises avant de commencer le travail sur le terrain. Toutefois, la plupart des choix résulteront des conditions mêmes du terrain : où regarder ? où et comment fouiller ? Qu'il s'agisse de reconnaissance en surface ou d'excavation, la cueillette et l'enregistrement des données doivent s'effectuer de manière consciencieuse et systématique. Car ce sont les uniques indices accessibles à l'archéologue pour identifier et expliquer les activités et les comportements culturels. Qui plus est, ce matériel fragile risque d'être détruit aussitôt qu'on le retire de sa matrice. A cause d'observations négligentes ou d'enregistrements erronés, les autres étapes de la démarche risquent d'être invalidées. C'est pourquoi l'archéologue dresse des cartes et des 
quadrillages, c'est pourquoi il note la position des vestiges avec tant de précision : il doit s'efforcer de rapporter au laboratoire une version aussi fidèle que possible des phénomènes détectés.

Avec le temps, les vestiges archéologiques se sont déposés dans un ordre qu'il s'agit de reconnaître. L'ordre de dépôt est établi grâce à l'étude de la stratification et au placement des témoins culturels dans chacune des strates identifiées. Idéalement, ce placement devrait s'effectuer aisément. Malheureusement, il arrive souvent que les strates aient été remaniées par des facteurs naturels (érosion, glissement de terrain, gel, lessivage) ou humains. Le but de l'excavation est d'observer comment se sont déposés et accumulés les vestiges. De plus, il s'agit de mettre en relation, pour chaque niveau culturel, les témoins qu'on a identifiés.

Tableau 4.2

Étapes logiques de l'enquête archéologique.

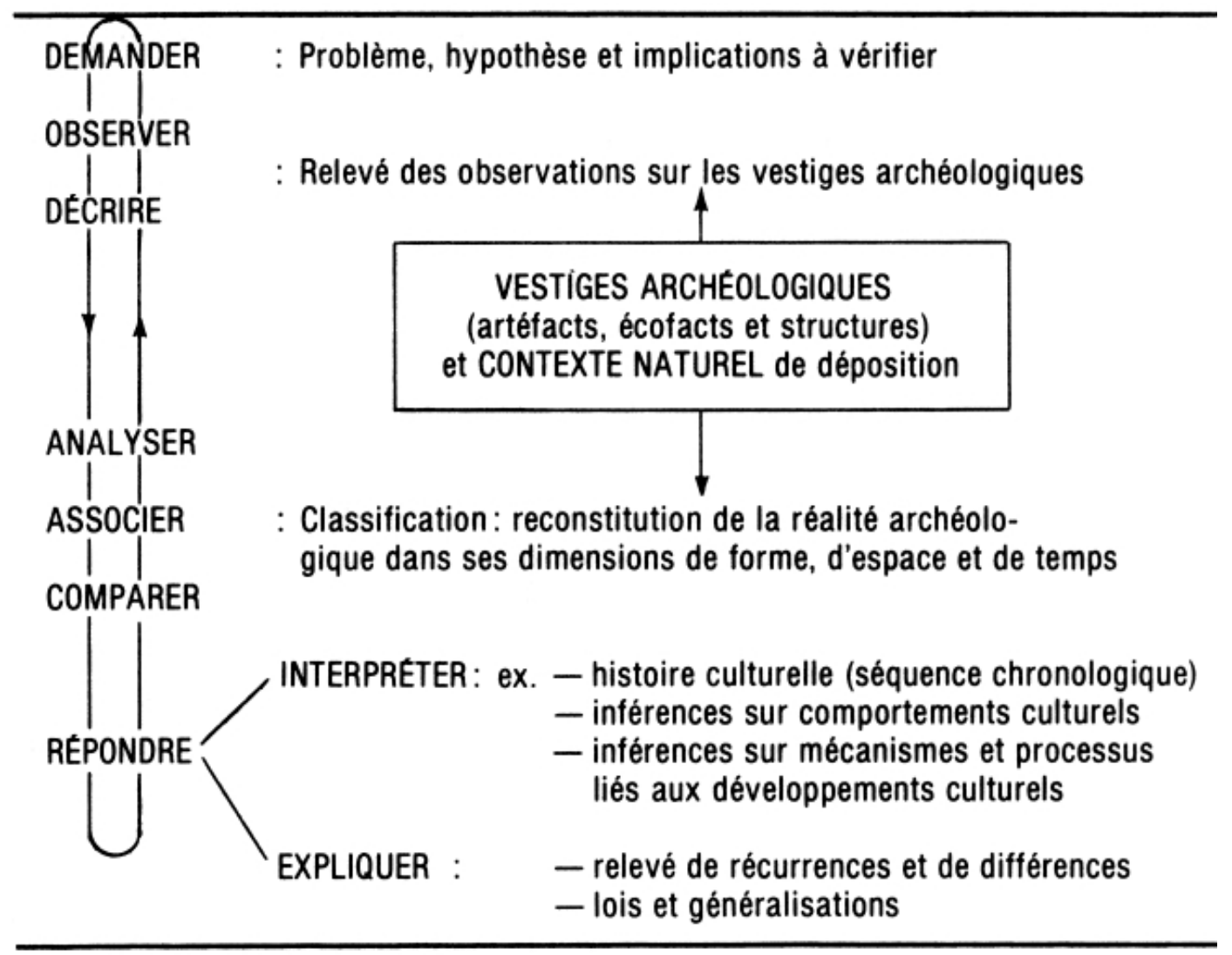


Les vestiges archéologiques ne sont pas toujours enfouis, et ceux qu'on trouve en surface, notamment les pyramides, les villes antiques ou autres structures demandent également à être analysés avec soin, afin d'en déterminer l'âge, le contenu, la fonction, les méthodes et, s'il y a lieu, les étapes de construction.

L'archéologue se spécialise dans la recherche et l'interprétation d'indices de comportements sociaux. Il doit néanmoins intégrer ceuxci dans le contexte naturel de leur époque, en raison de l'influence déterminante que ce dernier a pu exercer sur eux. L'étude de l'environnement, des sols, des animaux et des plantes - les écofacts - est aussi nécessaire que celle des artéfacts à l'interprétation des sociétés préhistoriques. L'archéologue s'en remet généralement à des spécialistes pour l'analyse de ces données. Les sols conservent, dans certaines conditions, des vestiges de plantes, du pollen, des ossements d'animaux. Ces restes, analysés par des zoologues, des botanistes et palynologues peuvent nous renseigner non seulement sur la composition de l'environnement, mais encore sur les conditions climatiques et leur changement à travers le temps.

La contribution des pétrologues et des géologues se rapporte principalement à l'étude de la nature des sols et de la composition des outils. Enfin, des physiciens et des chimistes aident l'archéologue à dater de façon précise les matériaux découverts. Par exemple, les analyses du charbon, trouvé en association avec des artéfacts, nous permettent de dater événements et activités préhistoriques.

Une fois les données recueillies, identifiées et enregistrées, il s'agit de les interpréter en fonction des problèmes que se pose l'archéologue. Une série d'instruments d'analyse ont été mis au point pour lui permettre d'accomplir cette tâche. En somme, comme dans tout travail scientifique, il s'agit de mettre de l'ordre dans le chaos, de classifier et de catégoriser le matériel. Il est impossible ici d'entrer dans les détails de la typologie et de rendre compte des problèmes et querelles qu'elle occasionne. Qu'il suffise de dire que les objectifs de la classification en archéologie sont multiples : on classifie les artéfacts en vue de les décrire, ou de les ordonner dans le temps ou encore de leur assigner une fonction. Traditionnellement, les archéologues se sont appliqués à 
identifier les artéfacts par catégorie (par exemple, outil, structure, céramique) et à utiliser chacune de ces catégories comme mesure de changement dans le temps. Les techniques de classification sont aujourd'hui fortement remises en question. D'une part, avec l'intérêt croissant pour la compréhension des comportements culturels, on a tendance à opter pour une typologie fonctionnelle et pour une analyse des relations entre les diverses catégories d'artéfacts. D'autre part, on applique de plus en plus les mathématiques, en particulier les statistiques et l'informatique, au traitement des données archéologiques, dans le but d'expliciter les procédures utilisées et d'éliminer l'élément trop souvent intuitif dans le choix des attributs de classification.

\section{Analyser, associer, interpréter, répondre}

\section{Retour à la table des matières}

L'archéologue s'est posé des questions ; il a maintenant à sa disposition un ensemble de données qui lui permettra peut-être d'y répondre. Cette étape est en pratique indissociable du reste de l'enquête archéologique. Mais le chercheur est maintenant en mesure de vérifier les hypothèses qu'il a formulées tout au long de son travail et, si elles s'avèrent exactes, de les élaborer davantage.

Il serait vain de songer à énumérer les formes de contribution de l'archéologie à la connaissance de l'homme et de son passé. Quitte à être taxée de réductionniste, je me limiterai à quelques généralisations.

L'archéologie interprète les phénomènes sociaux dans leur perspective diachronique autant que synchronique. En fait, la dimension temporelle la maintient dans une situation privilégiée par rapport aux autres disciplines anthropologiques. C'est en termes de milliers, si ce n'est de millions d'années, que pense l'archéologue. Alors que l'historien, le philosophe ou le sociologue des théories sociales spéculent le plus souvent à partir d'a priori non vérifiés, l'archéologue a la possibilité et même la responsabilité de documenter objectivement le changement social et culturel et d'en identifier les causes. 
Mais sur quoi, de façon plus particulière, nous informe l'archéologie ? Ses renseignements portent tout d'abord sur les populations humaines, sur leur histoire, leur évolution dans l'espace et dans le temps. Elle nous apprend ainsi que les groupes vivent et se perpétuent dans un milieu naturel qu'ils exploitent à diverses fins : les divers indices technologiques nous informent sur la nature et l'évolution de cette relation de l'homme avec son environnement. De plus, à l'intérieur de chacun de ces groupes s'établit tout un réseau d'interactions entre les individus de même que se produisent des interactions d'un groupe à l'autre. Ainsi la nature des relations intra et inter-groupes nous est révélée par des indices tels les coutumes funéraires, le degré de diversité à l'intérieur d'un site (notamment dans l'architecture), les réseaux d'échanges établis à partir de l'examen de la distribution de certains artéfacts, et l'iconographie.

Certains archéologues s'attachent davantage à l'étude de l'histoire culturelle d'une localité ou d'une région. La séquence chronologique du site permet tout d'abord d'ordonner dans le temps et dans l'espace les témoins des activités et de déceler, à travers les changements dans la culture matérielle, les transformations qui s'y sont produites. On peut ensuite appliquer cette démarche à toute une région ou à un continent. C'est ce qu'ont accompli les archéologues du début du siècle. D'autres préfèrent étudier la structure interne de la société préhistorique, c'est-à-dire mettre en relation les divers indices de comportements décelés dans un site ; en archéologie, on qualifie cette approche de contextuelle. Ainsi, on tente d'identifier le mode de subsistance, les activités et les comportements sociaux à une période donnée. A un niveau d'analyse plus général, certains archéologues se donnent pour tâche de déceler les processus et mécanismes de changement des sociétés préhistoriques. L'exemple le mieux connu est sans doute celui de la production alimentaire où, après s'être attardé à définir, théoriquement le plus souvent, ce mode de subsistance, on a tenté d'en identifier les premières manifestations. Puis on a ensuite voulu expliquer l'origine du développement de l'agriculture et de l'élevage dans ces sociétés et les formes que ces activités y ont prises. Les explications de ces phénomènes ont varié et continuent de varier : certaines privilégient des facteurs climatiques, d'autres, des facteurs démographiques comme déterminant majeur de l'adoption de ce nouveau mode de production. 
Il va sans dire que l'unanimité est loin d'exister chez les archéologues, tant au niveau théorique que méthodologique. La formation et les tendances théoriques de chacun d'eux influencent le plus souvent leur pratique scientifique et se traduisent par des orientations souvent divergentes et conflictuelles. En cela, l'archéologie ne diffère en rien des autres disciplines et serait même en excellente santé.

\section{Teotihuacán}

$\underline{\text { Retour à la table des matières }}$

J'aimerais en dernier lieu illustrer mon exposé sur la pratique de l'archéologie à l'aide d'un exemple tiré d'une société mésoaméricaine bien connue des archéologues.

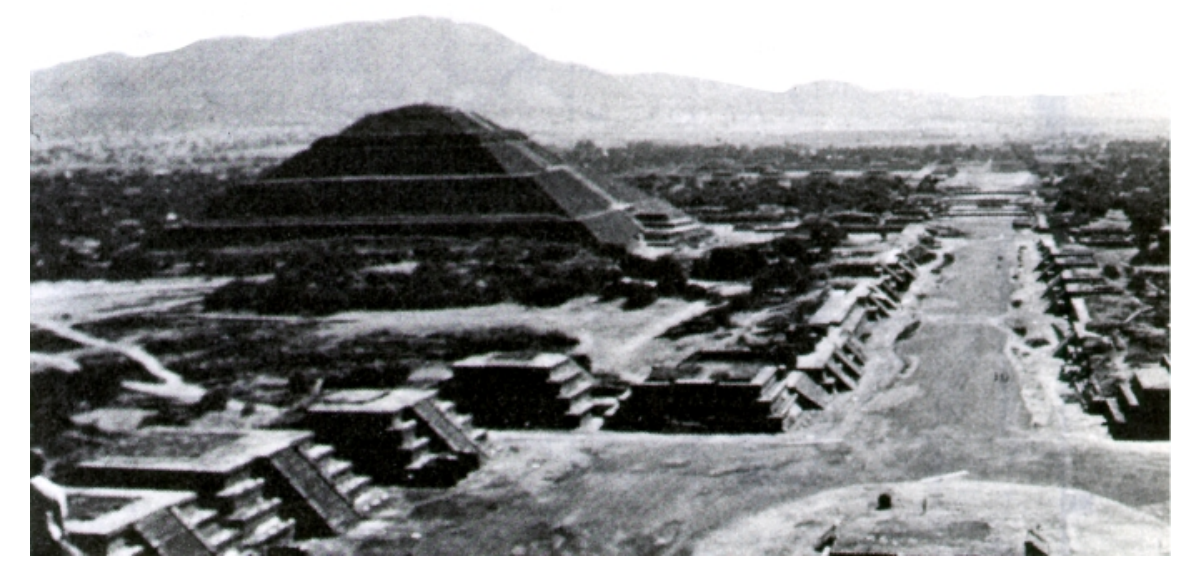

Figure 4.1

Teotihuacán : pyramide du soleil et avenue des Morts.

Les écrits du 16e siècle présentent Teotihuacán comme un site important où les dieux se seraient réunis pour planifier et réaliser la création du monde. Toutefois, la découverte par les archéologues de ce site, localisé au nord-est de la ville de Mexico, ne date que du siècle dernier. Des pyramides ont été retrouvées sous l'accumulation de terre qui en faisait une région de collines où on ne soupçonnait pas l'existence d'une cité. Des recherches ultérieures ont en effet permis d'iden- 
tifier une cité préhispanique, sans doute la plus ancienne des Amériques. À son apogée, qu'on établit vers l'an 450 ap. J.-C., cette cité s'étendait sur une superficie de $20 \mathrm{~km} 2$ et aurait abrité une population de 125000 à 200000 habitants.

Un projet intensif de reconnaissance du site archéologique, sous la direction de René Millon, a permis de dégager les diverses étapes de la construction de la ville ainsi que les conceptions architecturales et urbanistes de l'époque. La ville se divisait en quatre quadrants dont les axes principaux étaient constitués par l'avenue des Morts où se concentraient les édifices publics et religieux, parmi lesquels on peut mentionner les pyramides du soleil et de la lune, la citadelle et le palais de Quetzalpapalotl. Ces axes déterminaient le tracé des rues, qui adoptait une configuration géométrique et quadrillée similaire à celle des villes modernes.

L'étude des maisons et des résidences a fourni, à son tour, des informations sur la vie des habitants de la ville, leurs occupations et leur organisation économique et sociale. Ainsi, les résidences présentaient la forme de structures quadrangulaires et se composaient de nombreuses pièces communiquant par des portes et des corridors et donnant accès à une cour centrale. De plus, l'examen des concentrations d'artéfacts a permis d'identifier, à l'intérieur de la ville, des zones de spécialisation artisanale et économique : ateliers d'obsidienne (pour faire des outils) et de céramique, de travail lapidaire et de figurines. 


\section{Figure 4.2A}

Carte de Teotihuacan. Tiré de Urbanization at Teotihuacan, Mexico, v. 1, The Teotihuacan Map, 1973 by René Millon, tous droits réservés.

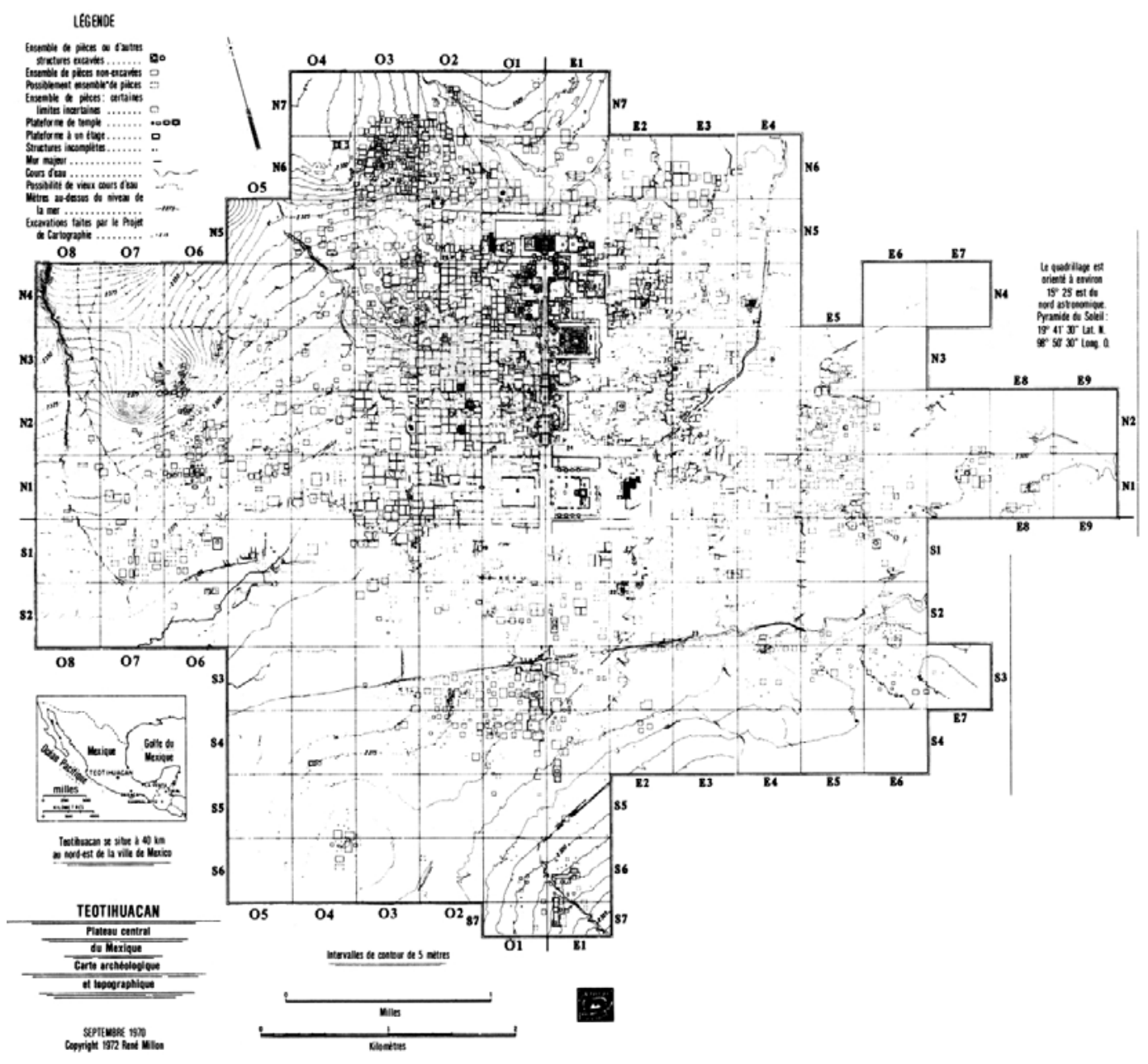

$\underline{\text { Retour à la table des matières }}$

La carte indique l'étendue de la ville vers 600 après J-C. : environ $20 \mathrm{~km} \mathrm{2.} \mathrm{On} \mathrm{y} \mathrm{représente} \mathrm{(1)} \mathrm{les} \mathrm{Structures} \mathrm{partiellement} \mathrm{ou} \mathrm{totale-}$ ment excavées, surtout le long de l'avenue des Morts (axe nord-sud), et (2) des reconstructions basées sur la reconnaissance des vestiges de surface de structures partiellement ou totalement excavées, faites par le Projet de Cartographie de Teotihuacán Un nombre indéterminé de structures, dans diverses parties de la ville, ont été ensevelies sous du 
limon ou ont été nivelées pur fins de production agricole. Remarquez la canalisation de la plupart des cours d'eau dans la vieille ville.

Projet de Cartographie de Teotihuacán

Directeur : René Millon

Département d'anthropologie

Université de Rochester

Rochester, New York

Subventionné par la National Science Foundation

Dessinateur ce chef : J. Armando Cerda

Principaux associés : Bruce Drewitt et George Cowgi

\section{SIGNIFICATION DES CHIFFRES}

Pyramide de la lune 1

Pyramide du soleil 2

Citadelle 3

Temple de Quetzalcoati 4

Avenue des Morts 5

Grand Ensemble 6

Avenue Ouest 7

Avoue Est 8

Barde des marchands

9

Tlamimilolpa 10

Xolalpan 11

Tepantitla 12

Murales des prêtres du maguey 13

Plaza 114

Maison des aigles 15

Vieille ville 16

Barrio de Oaxaca 17

Atetelco 18

La Ventilla A 19

La Ventilla B 21

La Ventilla C 21

Teopancaxco 22

Rio San Lorenzo

Rio San Juan 24

Réservoirs $\quad 25,26,27,51$ 
Acumulco 52

La quadrillage est orienté à environ $15^{\circ} 25^{\prime}$ est du nord astronomique.

Pyramide du Soleil : 19² 41' 30" Lat. N. 96 50' 30" Long. O.

Figure 4.2B

Carte détaillée

Carte de Teotihuacan. Tiré de Urbanization at Teotihuacan, Mexico, v. 1, The Teotihuacan Map, 1973 by René Millon, tous droits réservés.

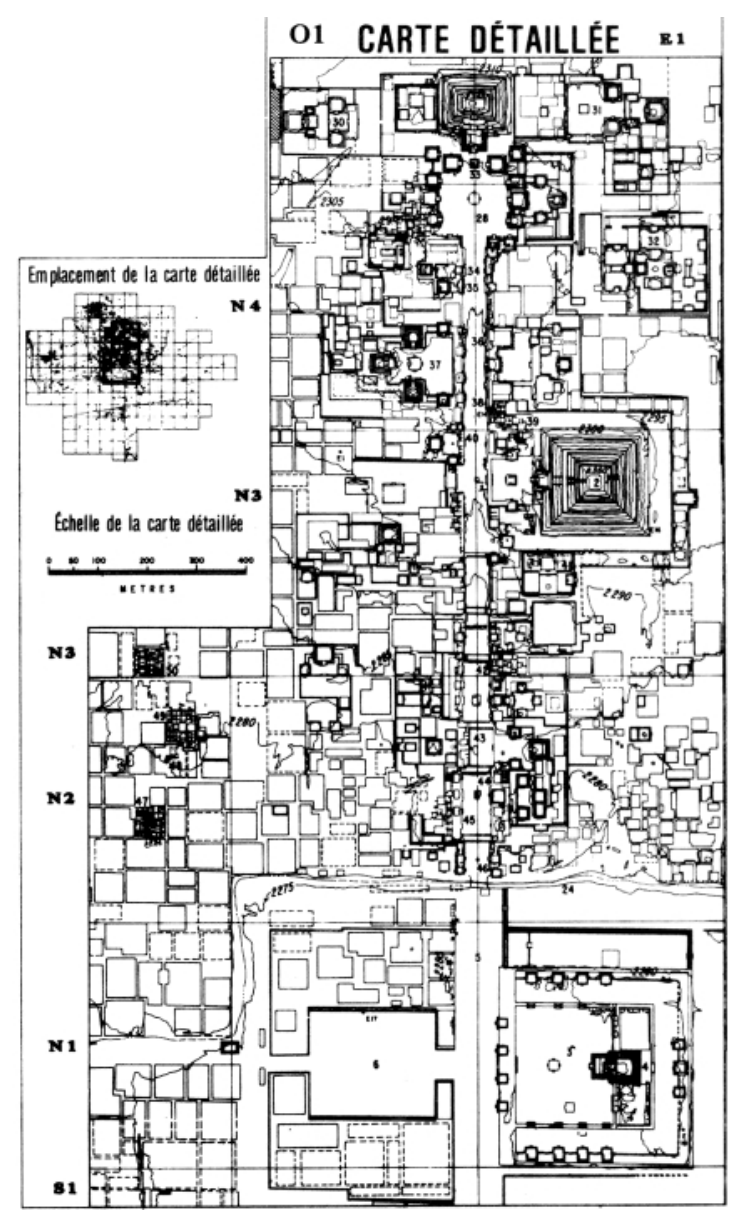

$\underline{\text { Retour à la table des matières }}$ 


\section{Légende de la carte détaillée}

Pyramide de la lune 1

Pyramide de soleil 2

Citadelle 3

Temple de Quetzalcoatl 4

Avenue des Morts 5

Gran Ensemble 6

Rivière San Juan 24

Place de la lune $\quad 28$

Palais de Quetzalpapaloti 29

Groupe 5' 30

Groupe 531

Ensemble Xala 32

Édifice du autels 33

Temple de l'agriculture $\quad 34$

Murales des animaux mythologiques 35

Murale du puma 36

Place des colonnes 37

Exploration de 189538

Palais du soleil 39

Patio des 4 petits temples 40

Maison des prêtres 41

Groupe Viking 42

Complexe de l'avenue des morts 43

Exploration de $1917 \quad 44$

Édifices surimposés 45

Explorations de 1908

Tititla 47

Patios de Zacuala $\quad 48$

Palais de Zacuala 49

Yayahuala 50 


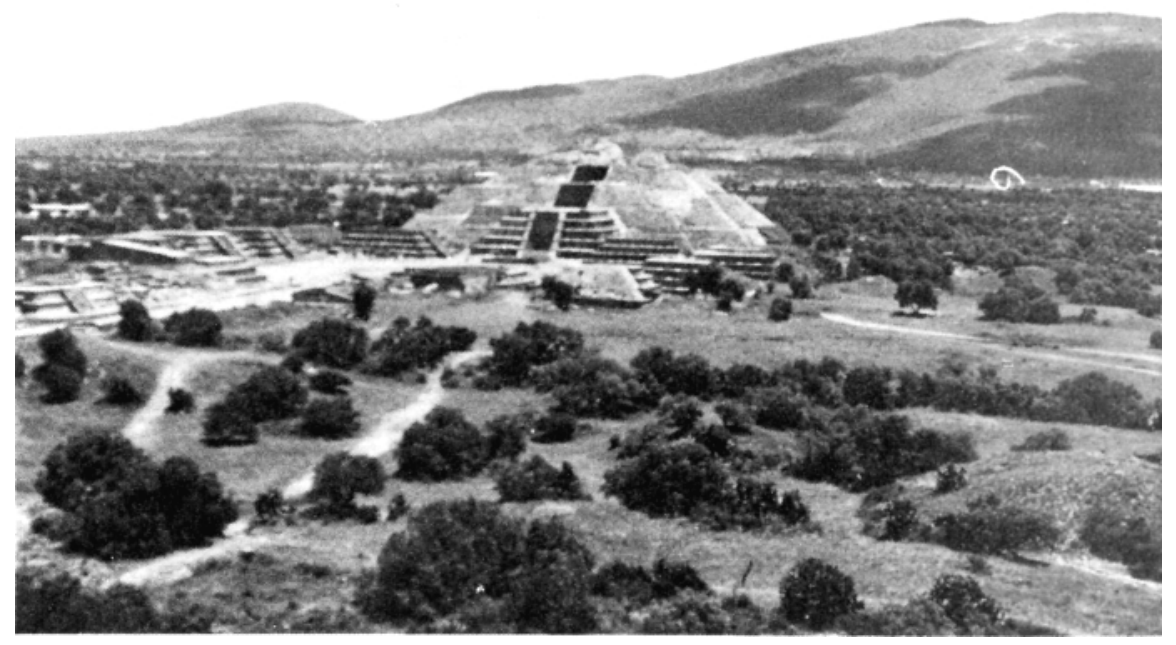

Figure 4.3

Teotihuacán : pyramide de la lune.

$\underline{\text { Retour à la table des matières }}$

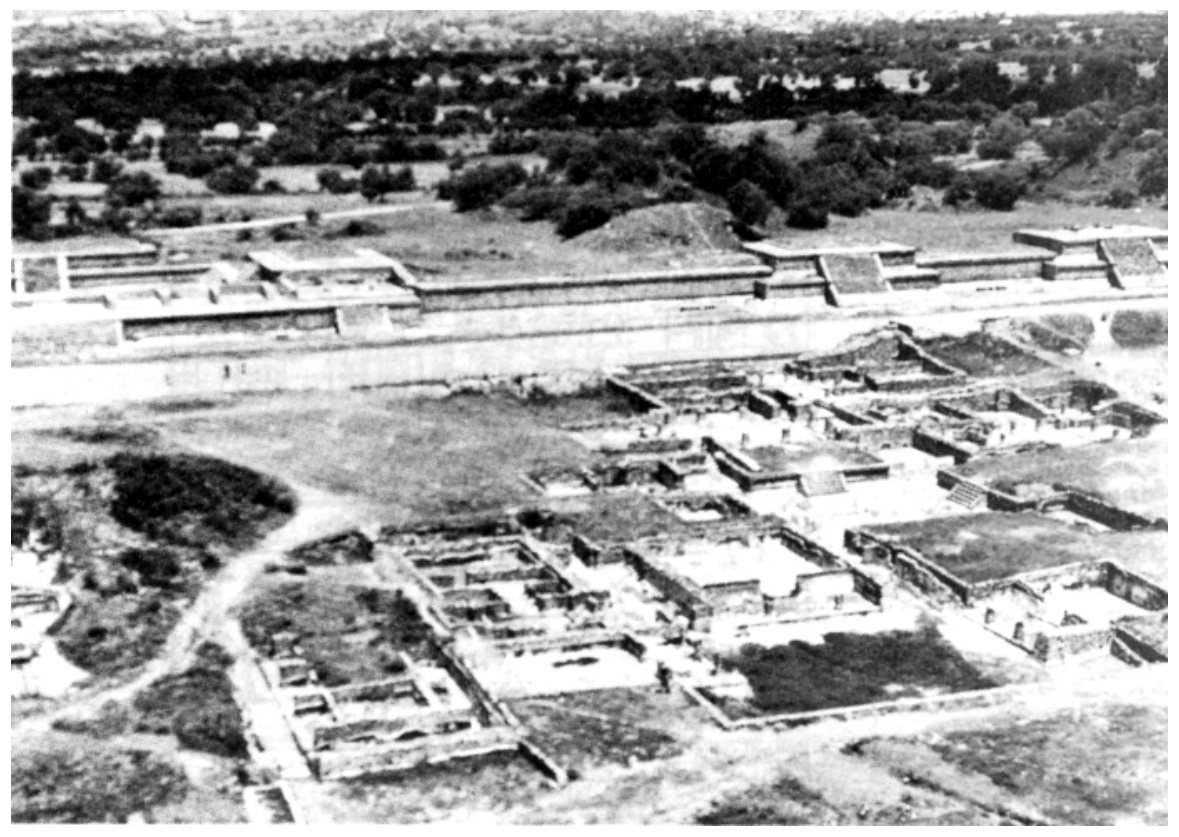

Figure 4.4

Teotihuacán : résidences près de la pyramide du soleil. 
L'étude combinée de l'architecture et de la céramique a fourni une chronologie détaillée des activités et des événements à Teotihuacán. Ces sources situeraient le début d'expansion de cette ville à quelques siècles avant J.-C., son apogée, vers l'an 450 ap. J.-C. et, finalement, son abandon, vers l'an 700 ap. J.-C. L'analyse stylistique de la céramique, des sculptures et des fresques murales a donné, à son tour, des indices de l'avancement technique et de l'idéologie des habitants de Teotihuacán.

Bien que notre connaissance de la ville même de Teotihuacán ait progressé, d'autres points demeurent encore dans l'ombre. Par exemple, comment et pourquoi Teotihuacán est-elle née et s'est-elle développée ? Comment sa population de 200000 habitants était-elle organisée sur le plan de la subsistance et de la production ? Quel rôle Teotihuacán a-t-elle joué aux niveaux économique, politique et religieux en Mésoamérique ? S'agissait-il d'un État ou d'un empire précolombien ? L'étude des régions avoisinantes de la ville, des indices de relations entre les diverses régions de la Mésoamérique (céramique, obsidienne) à la même époque, a contribué à donner des éléments de réponse à ces questions. Par exemple, l'analyse des sources d'obsidienne servant à faire des outils (on ne connaissait pas le métal au Mexique à cette époque) a permis de reconstituer un vaste réseau d'échanges économiques dont Teotihuacán était le centre. Certains voient même là une des causes du développement de la ville et de son contrôle sur le reste de la Mésoamérique. 


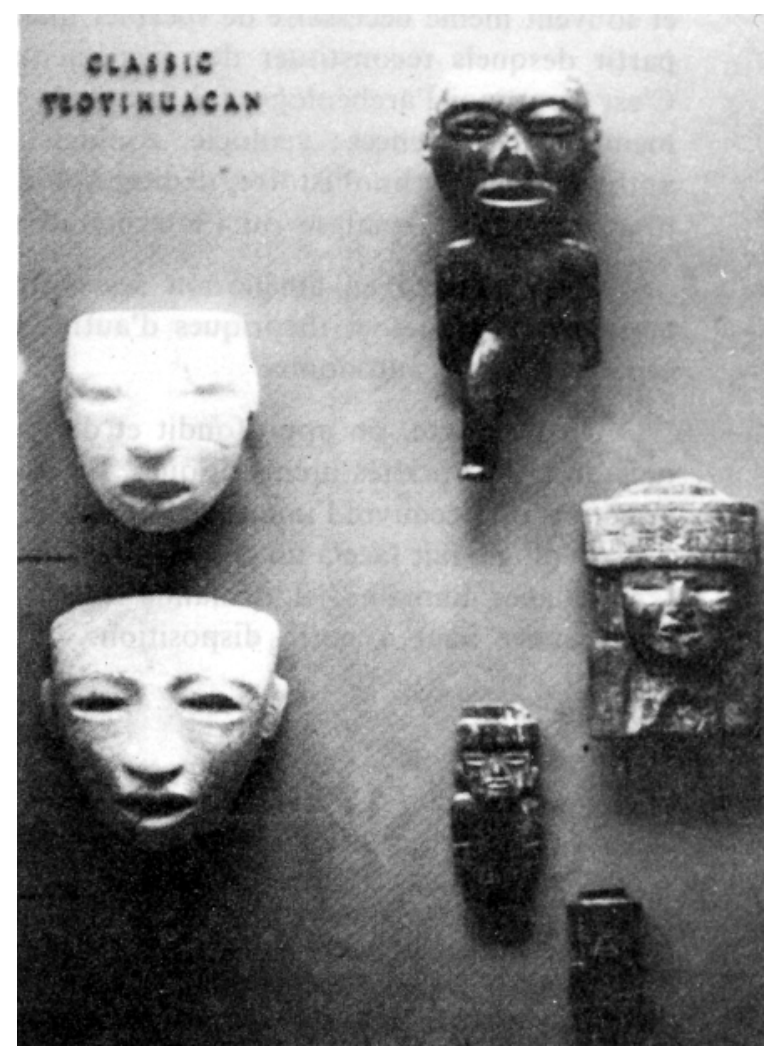

Figure 4.5

Teotihuacan : masques et figurines de pierre.

(American Museum of Natural History).

$\underline{\text { Retour à la table des matières }}$

Cet exemple, bien que présenté très sommairement, aura fait ressortir avec clarté, je l'espère, les possibilités et les limites d'interprétation qu'offrent à l'archéologie les témoins matériels de comportements sociaux. 


\section{Conclusion}

$\underline{\text { Retour à la table des matières }}$

L'archéologie est une discipline jeune : elle commence à faire ses preuves, elle est pleine de promesses, mais elle n'est pas autonome.

Son objet d'étude est aussi fascinant que varié : connaître et comprendre, à travers l'étude des vestiges qu'elles nous ont laissés, les sociétés qui nous ont précédés et qui ont, d'une façon ou d'une autre, déterminé ce que nous sommes aujourd'hui.

Et nous voilà entraînés dans le temps et dans l'espace, dans l'exploration d'un éventail de problèmes et souvent de mystères qu'il s'agit de résoudre systématiquement. Les théories à la Van Denneken (Chariots of the Gods) ${ }^{6}$ ne comptent pas d'adhérents parmi les archéologues. C'est par le biais d'un cadre théorique et méthodologique rigoureux que l'archéologie tente de résoudre ces problèmes.

L'archéologie est très souvent frustrée toutefois. Les données sur lesquelles reposent ses interprétations sont limitées et fragmentaires. Il lui est facile et souvent même nécessaire de spéculer quand elle n'a que quelques indices à partir desquels reconstituer des événements et des comportements sociaux. C'est pourquoi l'archéologie fait usage de tout ce qui peut contribuer à alimenter ses inférences ; géologie, zoologie, botanique, science économique, anthropologie, ethnohistoire, démographie, mathématiques viennent tour à tour compléter l'analyse ou l'interprétation des vestiges archéologiques.

$6 \quad$ VAN DENNEKEN : écrivain contemporain qui se livre à des spéculations hasardeuses et souvent malhonnêtes en s'appuyant sur des documents archéologiques. Il voit, entre autres, des astronautes sur certaines stèles mayas et des restes de pistes d'atterrissage construites par les habitants d'une autre planète, au Pérou et en Bolivie. 
C'est d'abord en améliorant ses outils d'analyse et en y intégrant les apports techniques et théoriques d'autres disciplines que l'archéologie peut espérer devenir autonome.

On découvre, on approfondit et d'autres sciences contribuent à la connaissance des sociétés préhistoriques. En même temps que notre connaissance s'accroît, on découvre l'immensité et la complexité du domaine qu'on aborde. L'archéologie fait face à un défi ; il s'agit de le relever. Le passé est là, riche en témoignages humains ; il demande à être reconstruit et les outils pour le comprendre sont à notre disposition.

\section{Bibliographie}

$\underline{\text { Retour à la table des matières }}$

Parmi les nombreux ouvrages d'introduction à l'archéologie, j'en ai retenu trois qui, par leur orientation et leur contenu, donnent une idée plus précise de ce qu'est et de ce que devient l'archéologie.

DEETZ, J.F., (1967). Invitation to Archaeology. New York, The Natural History Press. Courte introduction. Perspective théorique contemporaine.

FLANNERY, K.V., (ed.) (1976), The Early Mesoamerican Village. New York, Seminar Press. Théorie et pratique de l'archéologie. Discussion autour de l'étude d'un cas précis.

MOBERG, Carl-Axel, (1976). Introduction à l'archéologie. Paris, François Maspéro, Textes à l'appui. Excellente introduction pour les débutants comme pour les autres. 